et micrococciques frais mélangés avec 40 à $60 \mathrm{~cm}^{3}$ de lait et injectés trois à cinq fois avec cinq à sept jours d'intervalle et répétés, si nécessaire, deux à trois fois par année, nous avons obtenu des résultats bien manifestes, et dans la guérison et dans l'amélioration de la maladie.

\title{
LA CORROSION DES MÉTAUX PAR LE LAIT (1)
}

\author{
par \\ Colin G. FINK et Frederick A. ROHRMAN \\ de la Division d'Electrochimie de Columbia University (New-York).
}

\section{Introduction.}

Le lait est la plus importante des denrées alimentaires servant à la consommation de l'homme. Selon Houm [1], la production de lait aux Etats-Unis fut de 53.000.000 de tonnes en 1926, ayant une valeur marchande d'environ 4.000.000.000 de dollars. Lorsqu'on fait la comparaison avec d'autres industries, on constate que pour l'industrie automobile cette valeur atteint environ 3.500.000.000 de dollars, et pour l'industrie de l'acier, environ 3.000 .000 .000 de dollars.

Les dépôts coopératifs de lait, les usines de pasteurisation et le commerce ont exigé d'importantes installations d'appareils en métal. Une très importante partie du lait produit actuellement en Amérique est pasteurisée dans des appareils métalliques.

En contraste frappant avec son action, presque nulle, sur les organes du corps humain, l'effet du lait sur la plupart des métaux est très prononcé ; en fait, l'attaque des métaux par le lait est un des problèmes de corrosion les plus importants que devront résoudre la civilisation moderne et l'industrie.

\section{IMPORTANCE DE LA CORROSION PAR LE LAIT}

La corrosion des métaux par le lait ne devient pas seulement un problème économique à cause de son importance pour le renouvellement du matériel, mais également par la question de la valeur marchande des produits lactés et les facteurs physiologiques $q u$ 'elle influence. Un outillage défectueux peut être cause de la présence dans le lait de produits de la corrosion, dans des proportions telles, que, non seulement la saveur, mais également la valeur nutritive est altérée. La saveur peut être altérée uniquement par la solution de métal, d'où le goût métallique caractéristique, ou par l'action indirecte des métaux dissous sur les microorganismes du lait, ayant pour résultat la saveur « farineuse » [2].

(1) Journal of Dairy Science, vol. XV, $n^{\circ} 1$, janvier 1932, p. 73-86. 
On a obtenu de nombreuses données concernant l'effet des métaux se trouvant dans les aliments sur les animaux et sur les êtres humains. Dans ce mémoire, nous n'avons pas l'intention d'examiner l'aspect physiologique de la question. Cependant, ceci peut en être dit : la nature nous a donné le lait fomme un aliment qui n'a pas changé au cours de milliers d'années; on peut difficilement s'attendre, malgré les données d'expériences récentes, indiquant le contraire pour le fer et le cuivre, à ce que l'addition de métaux étrangers en solution dans le lait, à la suite de la corrosion de l'outillage, améliore le produit. Ne paraît-il pas logique que du lait qui a été pasteurisé dans des appareils de métal bien conditionnés et dont la teneur primitive en constituants métalliques et autres a été maintenue, sans qu'il y ait eu addition de substances étrangères, soit supérieur à du lait dont une partie des constituants métalliques a été éliminée, ou auquel des métaux étrangers ont été additionnés?

\section{RECHERCHES ANTÉRIEURES}

En aucune façon l'action corrosive du lait sur les métaux ne constitue un nouveau champ d'études. On sait depuis longtemps, que lorsque du lait est en contact avec du fer et du cuivre, non seulement sa saveur devient métallique, mais qu'en plus, il corrode rapidement ces métaux.

Supplex et Bexurs [3] ont constaté que la présence de quelques joints de bronze dans un appareil de pasteurisation avait pour effet une augmentation de la teneur en cuivre du lait. L'action du lait sur le cuivre et sur d'autres métaux a été étudiée par DoNAUER [4], Rioe et Miscall [5], Quam, Soloman et Hellwig [6], Miscall, Cavanaugh et Carodemus [7].

Le nickel et le métal Monel ont été étudiés par McKay, Fraser et Searlti [8], Flowers [9] et d'autres. Chiara [10] a étudiéces métaux en même temps que leurs alliages avec le zinc.

Hunziker, Cordes et Nissen [11] ont fait une étude très détaillée de l'action du lait sur une série de métaux et d'alliages. Ces auteurs et d'autres ont fait des observations sur le cuivre, le nickel, l'aluminium, le fer, l'étain, le zinc, le Monel (alliage de nickel, cuivre et fer), les alliages maillechort ou nickel-argent (German or nickel silver alloys), les alliages "inoxydables » (《 stainless ) alloys), etc.

\section{MÉTHODES D'OBSERVATION}

Presque tous les auteurs ont déterminé la corrosion des métaux par le lait en soumettant à l'action de celui-ci, pendant des périodes de temps bien définies, à certaines températures, des échantillons de métaux à poids connu. Après la période de contact, l'échantillon de 
métal était pesé de nouveau et le degré de corrosion était estimé d'après la perte de poids. Nous démontrerons plus loin - et des auteurs qui ont fait des recherches sur la corrosion l'ont mentionné - que la perte de poids n'est pas toujours une indication correcte 'du degré de corrosion; cependant, l'inexactitude des données d'une détermination de la perte de poids peut être corrigée en grande partie par des observations macroscopiques et microscopiques.

La très grande majorité des premiers observateurs fut unanime pour attribuer la corrosion des métaux par le lait à la corrosion par l'acide, e'est-à-dire à la substitution d'hydrogène à du métal.

\section{L'UTILISATION DES MÉTAUX DANS LES APPAREILS DE LAITERIE}

A la suite de leur étude détaillée récente, Hunziker, Cordes et Nissen [11] classent les métaux qu'ils ont étudiés dans les quatre groupes suivants, selon leur mérite relatif :

1. Le métal Allegheny, l'étain et le cuivre fortement étamé.

(Ces métaux n'ont pas d'effet sur la saveur, et il a été démontré qu'ils offrent la résistance maximum à la corrosion et au ternissement. La couche d'étain sur le cuivre doit être épaisse et intacte.)

2. Le nickel, l'aluminium et l'alliage manganèse-aluminium.

(Ces métaux ne donnent pas satisfaction entière dans des produits lactés à degré élevé d'acidité. En plus, le nickel ternit rapidement et l'aluminium est attaqué par les poudres alcalines utilisées pour le lavage.)

3. Métal Monel, Enduro, Ascoloy et nickel-argent.

(Les produits lactés ternissent appréciablement le métal Monel. Au cours de ces expériences, ce métal n'eut qu'une légère influence sur la saveur. L'Enduro et l'Ascoloy sont attaqués par les produits lactés, et on ne peut prévoir quelle sera leur résistance dans les appareils de laiterie. Le nickelargent ternit fortement et altéra la saveur de la majorité des produits lactés.)

4. Le fer étamé, le cuivre, le fer galvanisé, le fer et le zinc.

(Exception faite pour le fer fortement étamé, ce groupe ne peut convenir pour les appareils dans lesquels les produits lactés sont en contact avec le métal. Le fer étamé présente le même inconvénient lorsque la couche d'étain offre des solutions de continuité.)

Cette classification ne concorde pas complètement avec celle que nous avons adoptée à la suite des résultats que nous avons obtenus, plus particulièrement pour les alliages 18-8 (chrome- 
nickel-fer). Nous classons les métaux et alliages comme il suit (voir le tableau 2) :

(1) Alliages 18-8 (métal Allegheny, Enduro KA $\mathrm{A}_{2}$, ete.), hyblum, étain, cuivre fortement étamé et le cuivre ou le nicke] plaqué de chrome.

(2) Alliage $95 \mathrm{Cu}-5 \mathrm{Ni}$, fers chromés 12-13\%.

(3) Monel, les nickels-argents, le nickel, le cuivre, l'aluminium, le manganèse, l'alliage silicon-cuivre, l'A mbrac.

(4) Le fer, le fer étamé, le fer galvanisé, le zinc.

La description de nos expériences dans ce mémoire motivera notre classification.

\section{LA NATURE DE LA CORROSION}

Avant d'examiner la nature de la corrosion par le lait, il paraît logique de comprendre d'abord les principes fondamentaux de la corrosion par des solutions simples. Actuellement, la théorie électrochimique de la corrosion est pour ainsi dire universellement admise. Lorsqu'un métal entre en solution, on peut dire qu'on se trouve en présence du résultat d'une action électrochimique. Quand le métal entre en solution, sa charge, qui est zéro à l'état métallique, devient positive; il se charge d'ions positifs, et une quantité équivalente d'électricité doit passer de la solution au métal, de façon à neutraliser la charge. Dans le cas de corrosion par un acide, on peut exprimer cette modification de la façon suivante :

$$
\mathrm{MO}^{\mathrm{O}}+\mathrm{H}^{+} \mathrm{Cl}^{-} \rightarrow \mathrm{H}^{\circ}+\mathrm{M}^{+} \mathrm{Cl}^{-}
$$

La corrosion par des sels métalliques peut être exprimée comme il suit :

$$
\begin{gathered}
\mathrm{M}^{0}+\mathrm{Cu}^{+}+\mathrm{SO}^{4}=\rightarrow \mathrm{Cu}^{0}+\mathrm{M}^{+}+\mathrm{SO}^{4} \\
2 \mathrm{M}^{0}+\mathrm{Cu}^{+}+\mathrm{SO}^{4}=\rightarrow \mathrm{Cu}^{0}+\mathrm{M}^{2+} \mathrm{SO}^{4}=
\end{gathered}
$$

Dans le cas de corrosion due à la présence d'oxygène, on peut l'exprimer, pour le cuivre, comme il suit :

$$
2 \mathrm{Cu} \text { (métal) }+2 \mathrm{O}=2 \mathrm{CuO}
$$

puis :

$$
2 \mathrm{CuO}+2 \mathrm{HCl} \rightarrow \mathrm{CuCl}^{2}+\mathrm{H}^{2} \mathrm{O}
$$

Avant que les réactions (1) et (2) puissent se produire, les concentrations d'acide et de sels métalliques doivent être assez grandes par l'équation de Nernst pour rendre la réaction possible ; donc, à des concentrations peu élevées d'acide ou de sel métallique, la corrosion par ces facteurs peut ne pas être possible. En plus, pour obtenir les conditions du cas (1), de l'hydrogène doit, ou se dégager ou être éliminé avec de l'oxygène, condition sans laquelle la corrosion ne peut se produire. C'est en somme le phénomène bien connu de la polarisation par survoltage et l'oxygène agit comme dépolarisateur. 
Les cas qui viennent d'être exposés sont influencés par nombre de facteurs, qui déterminent la vitesse à laquelle ils progressent, tels : les caractéristiques chimiques et physiques du métal, le genre de solvant, la circulation, etc.

Il importe pourtant de retenir que l'oxygène peut parfois, à l'encontre de ce qui est généralement admis, retarder plutôt que favoriser la corrosion. Ainsi, lorsque la fourniture d'oxygène est assez abondante pour permettre la formation de couches d'oxyde légèrement solubles, on dit que le métal devient passif et qu'il résiste à la solution. Ceci est fréquemment le eas pour le fer et le nickel, ainsi que l'ont démontré les recherches d'Evans [12].

Tout problème de corrosion est un problème en lui-même souvent en contradiction apparente avec les conceptions fondamentales basées sur les données d'autres expériences. Il en est ainsi de la corrosion des métaux par le lait. On n'a jusqu'ici pu expliquer d'une façon satisfaisante pourquoi le lait avait une action si prononcée sur les métaux.

\section{LA NATURE DE LA CORROSION PAR LE LAIT}

On sait que le lait est une substance complexe contenant en même temps des radicaux organiques et inorganiques. Le lait ordinaire tend à être légèrement acide, ayant, à la température ordinaire, un $p H$ de 6,6-6,7 lorsqu'il est frais, de 4,6 lorsqu'il est acide. Il est alors évident que si on ne prend pas des précautions méticuleuses, dès que la traite est faite, le lait deviendra légèrement acide, grâce à l'acide lactique produit par les bactéries [13]. La hausse de la température du lait augmente l'acidité [14]; il est alors possible que ce facteur soit cause de l'accentuation de la corrosion des métaux par le lait. QUAM [15] et d'autres auteurs ont constaté que l'action corrosive du lait sur les métaux atteint son maximum à une température d'environ $80^{\circ} \mathrm{C}$., lorsqu'on augmente graduellement la température.

Il est nettement évident que l'acidité du lait n'est pas assez prononcée pour permettre la réaction exprimée par l'équation (1) lorsqu'il n'y a pas présence d'oxygène. Ce fait est démontré par les résultats obtenus par EMERY et HENLex [16], qui ont constaté que lors d'absence d'oxygène, le lait n'a qu'une influence très légère sur certains métaux. Lorsqu'on augmente la température du lait, sa teneur en oxygène diminue, et son acidité, ainsi qu'il a été dit déjà, augmente, et en même temps sa viscosité s'accentue [17]. Le premier facteur n'étant pas favorable à la corrosion au delà de $80^{\circ}$ C., il doit prédominer sur l'influence favorable de la viscosité et du $p H$, et provoquer une chute de la courbe du degré de solubilité. Il est bien connu qu'au delà de $80^{\circ} \mathrm{C}$., la teneur en oxygène de l'eau, 
laquelle eau peut être comparée au lait, diminue presque jusqu'à zéro.

La présence de grandes quantités d'oxygène dissous, à une température peu élevée, peut même avoir une influence inhibitrice sur la corrosion de métaux comme le nickel, sur lesquels se forment rapidement des eouches d'oxyde protectrices. Ceci peut être une explication du fait, que généralement dans l'unité de pasteurisation en nickel, les sections à basse température ne présentent que peu de corrosion, tandis que l'action corrosive se manifeste fréquemment dans le régénérateur, où une grande quantité d'oxygène est chassée de la solution et où il n'en reste qu'une quantité insuffisante pour permettre la formation d'une couche passive d'oxyde.

Donader [4] a établi, par calcul, que la rapidité de la corrosion est dix fois plus grande à la température de pasteurisation qu'à la température ordinaire. Il est admissible que la présence d'une certaine quantité d'oxygène soit nécessaire pour le type de corrosion exprimé par l'équation (1), mais il est très possible que la présence d'un excès d'oxygène soit inhibitrice de la corrosion de métaux comme le nickel.

\section{LA THÉORIE DE FINK SUR LA CORROSION DES MÉTAUX}

Frnk a attribué la corrosion du nickel par le lait au cuivre se trouvant dans ce lait [18]. C'est au cuivre présent dans tout lait normal qu'on doit attribuer une grande proportion de la corrosion observée. Nous pouvons exprimer la théorie des métaux-bases en nous servant de l'équation (2):

$$
\mathrm{Ni}+\mathrm{Cu}^{++} \rightarrow \mathrm{Ni}^{++}+\mathrm{Cu}
$$

Il est bien connu que tout lait normal contient du cuivre dans la proportion de 4 à 6 parties pour dix millions [19]. Cette teneur en cuivre augmente facilement lors de la présence de parties de bronze, de laiton ou de euivre dans les appareils qui sont en contact avec le lait. Dans ces cas, l'influence de l'oxygène est très réduite.

Le type de corrosion dû à la substitution des métaux est similaire à celui de la corrosion par les acides, dont il ne diffère que par l'influence d'un plus grand potentiel; en effet, dans des solutions équimoléculaires de cuivre et d'ions hydrogène, le nickel a une tendance plus prononcée, par environ 0 volt 3 , ̀̀ entrer en solution lorsqu'il est en présence de cuivre [20]. Evidemment, cette différence de potentiel est influencée par les concentrations d'activité respectives des ions hydrogène et du cuivre et peut, à $25^{\circ} \mathrm{C}$., être exprimée mathématiquement de la façon suivante :

$$
\mathrm{E}_{1}=\left(-, 23+\frac{0,059}{2} \log \mathrm{C}_{\mathrm{Ni}}\right)-\left(+, 34+\frac{0,059}{2} \log \mathrm{C}_{\mathrm{Cu}}\right)
$$




$$
\mathrm{E}_{2}=\left(-, 23+\frac{0,059}{2} \log \mathrm{C}_{\mathrm{Ni}}\right)-\left(0+0,059 \log \mathrm{C}_{\mathrm{H}}\right)
$$

On soustrait :

$$
\mathrm{E}_{3}=-, 34-\frac{0,059}{2}\left(\log \mathrm{C}_{\mathrm{Cu}}-2 \log \mathrm{C}_{\mathrm{H}}\right)
$$

Ces équations montrent théoriquement que la corrosion des appareils de pasteurisation en nickel est plus influencée par la concentration de cuivre dans le lait que par la concentration des ions hydrogène. En dehors de cela, l'hydrogène a un plus grand survoltage que le cuivre, ce qui donnerait encore une plus grande différence. On pourrait avancer que, dans le lait, le cuivre se trouve dans des combinaisons complexes et ne présenterait pas "l'activité " que manifestent les ions hydrogène. Nous démontrerons plus loin que cette hypothèse est insoutenable.

Riot et Miscall [5] ont démontré que lors d'écrémage de lait dans des écrémeuses de nickel, la teneur en cuivre diminuait. Cette élimination du cuivre n'aurait pu se faire si du nickel n'avait pas été dissous. Un examen méticuleux des parties en nickel de l'appareil qui sont en contact avec le lait démontra qu'elles présentaient une coloration foncée, qu'on ne parvenait pas à faire disparaître par le lavage. Nous servant d'une conduite de régénérateur dont la corrosion était très prononcée et dont la coloration noire avait été enlevée en la lavant au moyen d'acide nitrique, il nous fut possible de précipiter du cuivre métallique de-cette solution au moyen d'un courant électrique.

\section{Expérience sur grande échelle CORROSION DUE AU CUIVRE}

En possession de ces données, et ne perdant pas de vue les effets de la corrosion par les acides et de l'oxygène, une expérience fut faite dans une des usines de pasteurisation de la ville de New-York. Cette usine ressemblait à nombre d'usines de la ville ; le lait cru était conservé dans des réservoirs émaillés, les appareils de pasteurisation étaient en nickel et en cuivre et avaient les pièces de bronze généralement utilisées.

Dans cette usine, des échantillons de lait furent prélevés de treize parties différentes des appareils de pasteurisation. Ils le furent par une personne désintéressée, et ils furent numérotés : de cette façon nous n'avions d'autres indications que leurs numéros d'ordre, et nous n'avions aucun renseignement sur les échantillons (nous ne savions pas s'il y avait eu contact avec du métal chauffé ou non) et sur la nature du métal.

DÉTERMINATION DE LA PRÉSENCE DE CUIVRE DANS LES ECHANTILLons. - A ces échantillons de lait fut appliquée la méthode 
colorimétrique au xanthate. Préalablement, nombre d'essais avaient été faits avec des échantillons d'eau contenant des substances organiques, en vue de bien connaître les erreurs inhérentes à la méthode et de bien posséder sa technique. Des échantillons en double de $100 \mathrm{~cm}^{3}$ de lait furent évaporés lentement et calcinés dans des capsules de silice de $200 \mathrm{~cm}^{3}$. En vue de prévenir le contact possible de la flamme à gaz avec le contenu des capsules, le bord de celles-ci était garni d'une lame d'amiante.

La ealcination dans un four éleetrique fut essayée, mais les résultats obtenus furent irréguliers, à cause de la volatilisation du cuivre aux températures élevées. La calcination au four électrique donnerait sans doute des résultats satisfaisants si la température pouvait être maintenue relativement constante.

On observa très vite qu'à la surface de la silice, il se formait une croûte de coloration foncée, difficile à dissoudre. La dissolution de cette croûte fut obtenue dans de l'acide fluorhydrique; en conséquence, lorsqu'il fallut dissoudre les cendres après chaque calcination, quelques gouttes d'acide fluorhydrique furent additionnées à l'acide chlorhydrique. Dès ce stade, la méthode habituelle fut appliquée; en fait, évaporation de la dissolution dans l'acide chlorhydrique additionné d'eau, addition d'ammoniaque et neutralisation au même $p H$. Pour chaque échantillon de lait, les mêmes quantités d'eau, d'acide et d'ammoniaque furent utilisées, et le processus analy tique, la durée, la température furent aussi identiques que possible. Ces manipulations préliminaires furént suivies par l'application de la méthode eolorimétrique au xanthate, pour laquelle fut utilisée une échelle de couleurs bien connue. Toutes les comparaisons furent faites avec le même éclairage et dans les mêmes conditions de température.

Ce processus de détermination de la présence de cuivre, quoique excessivement laborieux, et exigeant de très grandes précautions, fournit des données très nettes et nous permit de déterminer si le lait avait passé sur du nickel, du cuivre ou un alliage de cuivre. Lorsqu'il avait passé sur du nickel, la teneur en cuivre diminua selon la réaction ( $2 a$ ), et lorsqu'il y avait eu contact avec du cuivre, la teneur en cuivre du lait augmenta selon la réaction (3). Les déterminations terminées, l'historique de chacun des échantillons de lait nous fut fourni, avee mention de tous les détails : température, contact métallique, etc. Pour chacun des treize échantillons, les teneurs en euivre déterminées concordèrent pour ainsi dire exactement avee celles qu'on aurait pu prévoir. Nous pouvions dire, d'après nos données d'analyse, si le lait venait de passer sur du cuivre et du nickel. Dans un cas seulement, lorsqu'il y avait eu contact avec du cuivre doublé de chrome, une légère déviation fut constatée, 
qui, en toute justice, pouvait encore être attribuée à une légère erreur expérimentale. Un exemple frappant fut fourni par les échantillons de lait provenant d'un des régénérateurs. Cet appareil était construit de la façon suivante : les 6 premières conduites étaient de nickel pur ; elles étaient suivies de 8 conduites de cuivre, puis encore de 16 conduites de nickel. A l'entrée du régénérateur, l'analyse du lait indiqua une teneur en cuivre de 0,43 p. p. m. (1). Après le passage par les six conduites de nickel pur, la teneur en cuivre était tombée à 0,31 p. p. m. ; à la sortie des huit conduites de cuivre, la teneur en cuivre était devenue 0,76 p. p. m. ; à la sortie des dernières conduites de nickel, la teneur en cuivre du lait était revenue à 0,43 p. p. m.

Ces données ne sont que des exemples démontrant à quel degré la teneur en cuivre du lait varie au cours de son passage par une unité de pasteurisation. Nombre des conduites de nickel présentèrent, par endroits, une corrosion très prononcée, indiquant qu'il y avait eu perte de nickel, et, bien que la teneur en nickel du lait n'ait pas été déterminée, on peut présumer, sans crainte de se tromper, que lors du passage dans l'unité de pasteurisation, la teneur en nickel du lait était accrue à cause de l'absence d'un métal moins noble sur lequel le nickel aurait pu se précipiter.

Sans aucun doute, les données de détermination du cuivre du lait varieront de jour en jour, selon l'activité de l'usine et les températures de pasteurisation, mais nous sommes persuadés que toujours leurs rapports ressembleront à ceux observés dans ce cas particulier.

(A suivre.)

\section{REVUE}

\section{LA NOUVELLE LOI SUR LES PRODUITS LAITIERS}

\section{par Maxime TOUBEAU}

Inspecteur général, chef de service au Ministère de l'Agriculture.

En adoptant, sans modifications, le projet de loi voté en 1932 par la Chambre pour la protection des produits laitiers, le Sénat a marqué sa volonté de défendre, même au prix de nouvelles dérogations au principe de la liberté du commerce, notre agriculture contre certaines entreprises industrielles jugées dangereuses pour elle (2).

Le fait est assez important pour qu'on s'y arrête et qu'on l'étudie, dans ses origines et dans ses conséquences.

C'est un texte déposé sur le bureau de la Chambre, le 11 juillet

(1) p. p. m. = partie(s) par million.

(2) Loi du 29 juin 1934. Le Lait, n० 139, p. 1027. 